\title{
Violência institucional referida pelo acompanhante da parturiente em maternidades públicas
}

Institutional violence reported by birth companions in public maternity hospitals Violencia institucional relatada por acompañante de parturienta en maternidades públicas

\author{
Lihsieh Marrero' ${ }^{1}$ iD https://orcid.org/0000-0002-2856-5682 \\ Odaléa Maria Brüggemann ${ }^{1}$ iD https://orcid.org/0000-0003-1210-6040 \\ Roberta Costa ${ }^{1}$ id https://orcid.org/0000-0001-6816-2047 \\ Carolina Frescura Junges ${ }^{1}$ io https://orcid.org/0000-0001-7337-9646 \\ Camilla Alexsandra Scheneck ${ }^{1}$ io https://orcid.org/0000-0001-8137-1871
}

Como citar:

Marrero L, Brüggemann OM, Costa R, Junges

CF, Scheneck CA. Violência institucional

referida pelo acompanhante da parturiente em maternidades públicas. Acta Paul Enferm. 2020;33:eAPE20190220.

DOI

http://dx.doi.org/10.37689/actaape/2020A002202

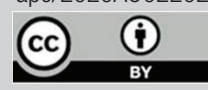

mais em exames físicos; Maternidades; Serviços de saúde; Violência; Violência contra a mulher; Gestantes

Keywords

Medical chaperones; Hospitals, maternity; Health services; Violence; Violence against women;

Pregnant women

Descriptores

Chaperones médicos; Maternidades; Servicios de salud Violencia; Violencia contra la mujer; Mujeres embarazadas

Submetido 29 de Julho de 2018

Aceito

21 de Outubro de 2019

\section{Autor correspondente \\ Lihsieh Marrero \\ E-mail: lihsiehm@gmail.com}

\section{Resumo}

Objetivo: Estimar a prevalência e os fatores associados à violência institucional contra a mulher durante 0 parto referida pelo acompanhante.

Método: Estudo transversal, realizado em três maternidades públicas da Região Metropolitana de Florianópolis, Santa Catarina, com 1.147 acompanhantes de parto. Os dados foram obtidos por meio de entrevista estruturada, no período de março de 2015 a maio de 2016. Na análise dos dados empregou-se regressão de Poisson simples e múltipla.

Resultados: A violência institucional contra a mulher foi relatada com maior frequência pelos acompanhantes do sexo masculino, que eram companheiro e/ou pai do bebê (74,7\%). Foi mencionado pelo menos um tipo de violência $(73,5 \%)$, sendo os tipos estrutural $(59,2 \%)$ e física $(31,4 \%)$ os mais prevalentes. Os fatores associados ao desfecho foram o parto vaginal, a termo, ocorrido entre terça e sextas-feiras e a maior escolaridade do acompanhante.

Conclusão: Os resultados desse estudo mostram que a presença do acompanhante não impede a ocorrência da violência institucional. As prevalências de violência estrutural, física, psicológica e verbal contra a mulher durante o parto, relatadas pelo acompanhante, apontam para a necessidade de mudanças macroestruturais, que garantam 0 atendimento livre de violências, com respeito ao protagonismo e aos direitos da mulher.

\section{Abstract}

Objective: To estimate the prevalence and the factors associated with institutional violence against women during hospitalization for delivery, as reported by companions.

Method: Cross-sectional study conducted in three public maternity hospitals in the metropolitan region of Florianópolis, Santa Catarina, with 1,147 birth companions. Data were obtained through structured interviews conducted from March 2015 to May 2016. Data analysis was performed using single and multiple Poisson regression.

Results: Institutional violence against women was more frequently reported by male companions, who were partners of the women and/or father of the baby (74.7\%). At least one type of violence was mentioned (73.5\%). Structural $(59.2 \%)$ and physical $(31.4 \%)$ violence were the most prevalent. The factors associated with the outcome were term vaginal deliveries, occurred between Tuesday and Friday, and higher level of education of the companion.

Conclusion: The results of this study show that the presence of the companion does not prevent the occurrence of institutional violence. The prevalence of structural, physical, psychological and verbal violence 
against women during childbirth, as reported by the companion, points to the need for macrostructural changes to ensure care free of violence, with respect to women's role and rights.

\section{Resumen}

Objetivo: Calcular la prevalencia y los factores relacionados con la violencia institucional contra la mujer durante el parto relatada por el acompañante.

Métodos: Estudio transversal, realizado en tres maternidades públicas de la Región Metropolitana de Florianópolis, estado de Santa Catarina, con 1.147 acompañantes de parto. Los datos se obtuvieron mediante entrevista estructurada, en el período de marzo de 2015 a mayo de 2016 . Para el análisis de los datos se empleó regresión de Poisson simple y múltiple.

Resultados: La violencia institucional contra la mujer fue relatada con mayor frecuencia por los acompañantes de sexo masculino, que eran el compañero y/o el padre del bebé (74,7\%). Se mencionó por lo menos un tipo de violencia (73,5\%) y las más prevalentes fueron la estructural (59,2\%) y la física (31,4\%). Otros factores relacionados con el desenlace fue el parto vaginal, a término, que ocurrió entre martes y viernes y una mayor escolaridad del acompañante.

Conclusión: Los resultados de este estudio demuestran que la presencia del acompañante no impide que ocurran episodios de violencia institucional. La prevalencia de violencia estructural, física, psicológica y verbal contra la mujer durante el parto, relatada por el acompañante, indica la necesidad de cambios macroestructurales que garanticen una atención sin violencia y con respeto al protagonismo y a los derechos de la mujer.

\section{Introdução}

A violência institucional (VI) resulta da desigualdade de poder na interação dos sujeitos em uma instituição. ${ }^{(1)}$ Nos serviços de atenção obstétrica, a VI é reconhecida em situaçóes que a estrutura organizacional e as condutas profissionais causam danos ou prejuízos à mulher, ao bebê e à família. ${ }^{(2)}$ Desrespeito e abuso de profissionais direcionados à parturiente, também conhecidos como violência obstétrica (VO), são manifestações da VI. ${ }^{(3-5)} \mathrm{No}$ entanto, a VO apresenta como característica a intencionalidade da ação, não havendo uma definição consensuada quanto a tipologia e aos critérios para sua identificação em diferentes contextos, ${ }^{(6,7)}$ justificando o uso do termo VI neste estudo.

Apesar dos progressos nas políticas de saúde, a VI contra a mulher durante a internação para o parto, permanece disfarçada nos fluxos assistenciais e com magnitude desconhecida. Estima-se que, aproximadamente, $25 \%$ das mulheres que tiveram partos em maternidades brasileiras nos últimos anos, sofreram algum tipo de violência, com maior prevalência entre as negras, de menor escolaridade, atendidas no setor público e sem acompanhante. ${ }^{(8,9)}$

No Brasil, a presença do acompanhante durante a internação para o parto é um direito previsto em lei, usufruído por menos de 30\% das parturientes. (4) Comumente, o acompanhante pertence à rede social da mulher e lhe provê conforto e segurança, contribuindo para o parto fisiológico e a sua satisfação. ${ }^{(10-14)}$ No parto institucionalizado, o acompanhante é um avaliador externo dos cuidados presta- dos, capaz de perceber a VI mesclada aos protocolos assistenciais durante a internação da mulher para o parto. ${ }^{(15-17)}$

Neste contexto, a investigação da VI contra a mulher durante a internação para o parto, a partir da perspectiva do acompanhante, contribui tanto como uma medida indireta quanto para a elaboração de estratégias de combate e prevenção do problema. Cabe destacar que, no Brasil, o acompanhante, independente do parentesco com a mulher, faz parte da sua rede de apoio, que no parto desempenha diversos papéis, entre eles o de controle social. O objetivo deste estudo foi estimar a prevalência e os fatores associados à VI contra a mulher durante a internação para o parto, referida pelo acompanhante em maternidades públicas da Região Metropolitana de Florianópolis (RMF), SC.

\section{Métodos}

Estudo transversal, inserido em um macroprojeto aprovado pelo Comitê de Ética em Pesquisa da Universidade Federal de Santa Catarina (parecer $n^{\circ}$. 541.296, CAEE 25589614.3.0000.0121), conduzido nas três maiores maternidades da RMF, referências regionais na atenção obstétrica e que permitirem a presença do acompanhante durante a internação da mulher para o parto, por isso eleitas para o estudo.

A população de estudo foram os acompanhantes de parto, sendo elegíveis aqueles que permaneceram ao lado da parturiente durante o trabalho de parto e 
parto. Foram excluídos os acompanhantes de mulheres que não falassem/compreendessem o idioma português, com gestação múltipla, submetidas à cesariana de urgência ou eletiva, situaçóes em que a mulher náo entrou em trabalho de parto ou quando ela ou o feto/recém-nascido evoluíram para o óbito.

O tamanho da amostra foi calculado, considerando como parâmetro o número de nascimentos em cada maternidade no ano anterior ao planejamento do estudo (2013), a prevalência presumida em 50\%, o nível de confiança de $95 \%$ e o erro máximo de $5 \%$. O tamanho de amostra calculado foi de 307 acompanhantes na Maternidade A, 349 na Maternidade B e 346 na Maternidade $C$, totalizando uma amostra mínima de 1.002 acompanhantes. ${ }^{(10)}$ Não foram utilizados métodos probabilísticos específicos para a seleção dos sujeitos, por se tratar de uma amostra intencional.

Do total de partos ocorridos nas maternidades estudadas durante a coleta de dados, foram identificados 4.299 acompanhantes, destes 4.004 eram elegíveis. Dentre os elegíveis, 2.541 não foram localizados pelos entrevistadores durante a internação, impossibilitando o convite para participar do estudo. Ao todo, 1.463 acompanhantes foram convidados, dos quais $289(20,1 \%)$ recusaram o convite, sendo o tempo de entrevista o motivo mais frequente. Ao final participaram do estudo 1.147 acompanhantes.

A coleta de dados foi conduzida entre março de 2015 e maio de 2016, por entrevista estruturada em formulário eletrônico, realizada individualmente com o acompanhante, durante a internação da mulher para o parto, em local reservado. A entrevista era composta por perguntas fechadas sobre as características sociodemográficas; experiência anterior em ser acompanhante de gestante/parturiente; participação do acompanhante no pré-natal, parto e pós-parto e satisfação com a experiência atual. A construção do instrumento foi baseada na experiência dos pesquisadores na atenção à saúde da mulher, na literatura sobre a inserção do acompanhante de parto institucionalizado e no inquérito nacional sobre parto e nascimento "Nascer no Brasil". (18)

Os dados coletados eram revisados diariamente, possibilitando a identificação e correção de inconsistências. ${ }^{(10)}$ Após o encerramento da coleta de dados, replicou-se, por contato telefônico, parte da entrevista em uma amostra aleatória de 5\% dos participantes de cada maternidade, para comparação de respostas das entrevistas. Não foram identificadas inconsistências.

Neste estudo, para a construção dos indicadores de VI utilizou-se quatro das sete categorias de desrespeito e abuso contra a mulher durante o atendimento obstétrico propostas por Bohren et al.: ${ }^{(3)}$ estrutural, verbal, física e psicológica. Assim, caso o acompanhante relatasse que a parturiente foi vítima de ao menos um dos quatro tipos de violência, considerou-se como caso ou desfecho de interesse. Para isso, utilizou-se um conjunto de nove perguntas acerca do atendimento recebido nas instituiçóes.

A VI estrutural foi considerada como o náo atendimento e/ou atendimento precário em decorrência da infraestrutura inadequada, falta de recursos humanos e materiais, indisponibilidade de leitos, imposição de rotinas institucionais que causam danos à parturiente. Para estimar sua frequência, perguntou-se: "os profissionais de saúde ofereceram líquidos e/ou alimentos para a mulher durante o trabalho de parto?", "o local em que a gestante vivenciou o trabalho de parto e o parto era adequado?", "havia sempre um profissional disponível para atender as demandas da mulher?", todas com respostas codificadas como "sim" ou "não". A resposta negativa a pelo menos uma dessas perguntas foi considerada como caso de violência estrutural.

A VI verbal, descrita como tratamento áspero, ameaças, repreensão, gritos e xingamentos por profissionais de saúde durante o atendimento ao parto, foi estimada a partir da pergunta "a mulher foi exposta a alguma situação de violência verbal?”, com resposta codificada como "sim" ou "não", sendo as respostas positivas consideradas como caso.

Empurróes, exames dolorosos e repetidos; procedimentos danosos à saúde, restrição de movimentos e obrigar a parturiente a manter posições não desejadas por ela são situaçóes consideradas VI física. Neste estudo, foi avaliada pelas perguntas: "a mulher foi exposta a alguma violência física?" e "a mulher assumiu a posição deitada com as pernas levantadas no momento do parto normal?”, com alternativas de resposta "sim" ou "não". As respostas positivas foram consideradas casos.

A VI psicológica é caracterizada por ameaças, negação de atendimento ou de método de alívio da dor, 
abandono de atendimento, humilhação intencional, constrangimento, imposição de decisóes profissionais, desqualificação da opinião da mulher, fornecimento de informaçôes duvidosas ou não informação, banalização/negligência do sofrimento ou necessidades da mulher. Considerou-se como desfecho para esse tipo de violência quando o acompanhante respondeu "sim" para a pergunta "a mulher foi exposta a alguma violência psicológica?” e/ou "não” para às perguntas "os profissionais explicaram para a gestante o que estava acontecendo durante o trabalho de parto?" e "as informações relacionadas ao andamento do trabalho de parto foram repassadas à mulher de forma clara e de fácil compreensão?”.

As variáveis selecionadas para caracterizar o parto em que o acompanhante esteve presente foram: via de nascimento (parto vaginal ou cirurgia cesárea), classificação do parto quanto à idade gestacional referida pelo acompanhante (a termo, prematuro), período da semana de ocorrência do parto (sábado, domingo e segunda-feira, terça a sexta-feira); turno do (dia [7:00-19:00h], noite [19:01-6:59h]) e tempo de permanência ao lado da parturiente da internação ao nascimento (em horas).

Para a caracterizaçáo sociodemográfica dos acompanhantes utilizou-se as variáveis sexo (masculino, feminino), idade (em anos), cor/raça autodeclarada (branca, preta/indígena, parda/amarela), escolaridade (em anos de estudo), estado civil (casado/união estável, solteiro/divorciado/viúvo), vínculo com a mulher (companheiro e pai do bebê, mãe/irmã/amiga), experiência anterior com o parto (sim, não), participação em curso de gestação/parto (sim, náo), participaçáo em palestra sobre gestação/parto (sim, não).

$\mathrm{Na}$ análise dos dados empregou-se regressão de Poisson simples e múltipla, com estimador de matriz de covariância consistente ${ }^{(19)}$ do tipo HC3, para estimar a razáo de prevalência $(\mathrm{RP})$ entre os acompanhantes que relataram qualquer tipo de VI contra a mulher durante a internação para o parto, em relação aos que não relataram. A análise de regressão foi efetuada em duas etapas. $\mathrm{Na}$ primeira, mediante regressão simples, a associação de cada covariável foi avaliada separadamente em relação ao desfecho, selecionando-se somente as covariáveis com $\mathrm{p}$-valor $<0,2$. Na segunda etapa, testou-se modelos de Poisson múltiplos. Após a obtenção do modelo final, foram testadas possíveis interações entre: relato de qualquer tipo de VI versus idade do acompanhante, relato de qualquer tipo de VI versus escolaridade do acompanhante e relato de qualquer tipo de VI versus classificação do parto.

Em todos os passos, o teste da razão de verossimilhança foi utilizado como critério de seleção de variáveis e dos modelos. Os intervalos de confiança para as RP do modelo final foram estimados ao nível de 95\%. Os dados foram analisados com auxílio do programa estatístico $R$, versão 3.3.2.

\section{Resultados}

A maioria dos entrevistados era do sexo masculino $(76,9 \%)$, autodeclarados de cor/raça branca $(53,8 \%)$; com mediana de idade de 30 anos (Intervalo interquartílico=24-37); com tempo mediano de estudo de 10 anos (Intervalo interquartílico=7-11) (dados não apresentados); casados/uniáo consensual $(79,7 \%)$; companheiro e pai do bebê $(76,7 \%)$, que permaneceram ao lado da parturiente por tempo mediano de oito horas (Intervalo interquartílico=4-13) e sem experiência anterior em acompanhar parto $(76,0 \%)$. Além disso, a maioria dos entrevistados náo havia participado de curso de parto $(96,9 \%)$ e nem de palestras sobre gestação/ parto (92,1\%). Predominaram acompanhantes de parto vaginal $(75,1 \%)$, a termo $(91,3 \%)$ ocorridos entre terça a sexta-feira $(60,6 \%)$ durante o dia $(54,6 \%)$ (Tabela 1$)$. Os relatos de VI contra a mulher durante a internação para o parto foram mais frequentes entre os acompanhantes homens (74,8\%), companheiro e/ou pai do bebê $(74,7 \%)$, que participaram de cursos de parto $(73,2 \%)$ e de palestras sobre gestação/parto (73,5\%), que acompanharam partos vaginais $(77,6 \%)$, a termo $(73,5 \%)$, ocorridos de terça à sexta-feira $(75,9 \%)$ e durante o período diurno (74,3\%) (Tabela 1).

$\mathrm{O}$ percentual de relato de pelo menos um tipo de VI foi de $73,5 \%$. A VI estrutural foi relatada por $59,2 \%$ dos acompanhantes, a VI física por 31,4\%, a violência psicológica por $15,9 \%$ e a VI verbal por $3,5 \%$ (Tabela 2). 
Tabela 1. Variáveis sociodemográficas do acompanhante e do atendimento, segundo o relato de violência contra a mulher durante a internação para o parto em maternidades públicas

\begin{tabular}{|c|c|c|c|}
\hline \multirow{2}{*}{ Variáveis } & \multicolumn{3}{|c|}{$\begin{array}{l}\text { Relato de violência institucional } \\
\text { contra mulher no parto }\end{array}$} \\
\hline & $\underset{\mathrm{n}(\%)}{\operatorname{Sim}}$ & $\begin{array}{l}\text { Não } \\
\mathrm{n}(\%)\end{array}$ & Total \\
\hline \multicolumn{4}{|l|}{ Sexo } \\
\hline Masculino & $658(74,8)$ & 222(25,2) & $880(76,9)$ \\
\hline Feminino & $183(69,3)$ & $81(30,7)$ & $264(23,1)$ \\
\hline \multicolumn{4}{|l|}{ Cor/Raça autodeclarada } \\
\hline Branca & $458(74,5)$ & $157(25,5)$ & $615(53,8)$ \\
\hline Preta/Parda & $312(72,9)$ & $116(27,1)$ & $428(37,4)$ \\
\hline Amarela/Indígena & $71(70,3)$ & $30(29,7)$ & $101(8,8)$ \\
\hline \multicolumn{4}{|l|}{ Estado civil } \\
\hline Casado/União consensual & $673(73,8)$ & 239(26,2) & $912(79,7)$ \\
\hline Solteiro/Divorciado/Viúvo & $168(72,4)$ & $64(27,6)$ & $232(20,3)$ \\
\hline \multicolumn{4}{|l|}{ Vínculo com a mulher } \\
\hline Companheiro e pai do bebê & $655(74,7)$ & $222(25,3)$ & $877(76,7)$ \\
\hline Mãe/Irmã/Amiga & $186(69,7)$ & $81(30,3)$ & $267(23,3)$ \\
\hline \multicolumn{4}{|c|}{ Experiência anterior em acompanhar parto } \\
\hline Não & $645(74,1)$ & $225(25,9)$ & $870(76,0)$ \\
\hline Sim & $196(71,5)$ & $78(28,5)$ & $274(24,0)$ \\
\hline \multicolumn{4}{|l|}{ Participação em curso de parto } \\
\hline Não & $812(73,2)$ & $287(26,8)$ & $1109(96,9)$ \\
\hline Sim & $29(82,9)$ & $6(17,1)$ & $35(3,1)$ \\
\hline \multicolumn{4}{|c|}{ Participação em palestra sobre gestação/parto } \\
\hline Não & $775(73,5)$ & $279(26,5)$ & $1054(92,1)$ \\
\hline $\mathrm{Sim}$ & $66(73,3)$ & $24(26,7)$ & $90(7,9)$ \\
\hline \multicolumn{4}{|l|}{ Via de nascimento } \\
\hline Parto Vaginal & $667(77,6)$ & $192(22,4)$ & $859(75,1)$ \\
\hline Cirurgia Cesárea & $174(61,1)$ & $111(38,9)$ & $285(24,9)$ \\
\hline \multicolumn{4}{|l|}{ Classificação do parto } \\
\hline Á termo & $767(73,5)$ & $277(66,5)$ & $1044(91,3)$ \\
\hline Prematuro & $74(74,0)$ & $26(26,0)$ & $100(8,7)$ \\
\hline \multicolumn{4}{|l|}{ Período da semana } \\
\hline Terça a sexta-feira & $526(75,9)$ & $167(24,1)$ & $693(60,6)$ \\
\hline Sábado a segunda-feira & $315(69,8)$ & $136(30,2)$ & $451(39,4)$ \\
\hline \multicolumn{4}{|l|}{ Turno do Parto } \\
\hline Dia & $463(74,3)$ & $160(25,9)$ & $623(54,6)$ \\
\hline Noite & $376(72,6)$ & $142(27,4)$ & $518(45,4)$ \\
\hline
\end{tabular}

Tabela 2. Prevalência de violência institucional contra a mulher durante a internação para o parto, segundo tipo de violência referida pelo acompanhante $(\mathrm{n}=889)$

\begin{tabular}{lcc}
\hline Tipos de violência & $\mathbf{n}(\%)$ & $\mathbf{I C}_{95 \%}$ \\
\hline Estrutural & $634(59,2)$ & $56,3-62,0$ \\
Física & $359(31,4)$ & $28,7-34,1$ \\
Psicológica & $182(15,9)$ & $13,8-18,0$ \\
Verbal & $40(3,5)$ & $2,4-4,6$ \\
\hline
\end{tabular}

$\mathrm{Na}$ análise de regressão simples as covariáveis período da semana, turno, sexo, cor/raça, estado civil, experiência anterior em ser acompanhante de parto, participação em curso de parto e em palestras sobre gestação/parto apresentaram valores de p>0,20. Já a covariável vínculo com a mulher mostrou-se associada ao desfecho, mas na seleção dos modelos foi removida. A covariável tempo de permanência ao lado da mulher (em horas) não esteve associada ao desfecho, mas foi mantida no modelo final para controle de confusão.

$\mathrm{Na}$ análise de regressão múltipla foram excluídos três casos que apresentaram dados faltantes nas variáveis consideradas para esta etapa $(n=1.144)$. No modelo final ajustado, os acompanhantes de parturientes que tiveram parto vaginal perceberam, em média, 1,48 vezes mais VI do que aqueles que acompanharam cirurgias cesáreas. $\mathrm{O}$ período da semana de ocorrência do parto, entre sábado e segunda-feira foi o momento em que o acompanhante percebeu, em média, 17\% menos VI, em relação aos demais dias da semana. A cada acréscimo unitário de escolaridade do acompanhante, a percepção de VI aumentou, em média, 2\%. Os acompanhantes de partos prematuros relataram, em média, 30\% menos VI, em relação aos acompanhantes de parto a termo (Tabela 3).

No modelo final, testou-se a interaçáo entre as covariáveis relato de qualquer tipo de VI versus idade do acompanhante; relato de qualquer tipo de VI versus escolaridade do acompanhante e relato de qualquer tipo de VI versus classificação do parto, todas com valor de p>0,10 (dados não apresentados em tabela).

Tabela 3. Razão de prevalência (RP) bruta e ajustada de violência institucional contra a mulher durante a internação para o parto percebida pelo acompanhante em maternidades $(n=1144)$

\begin{tabular}{|c|c|c|c|c|}
\hline Variáveis & $\mathrm{RP}^{*}\left(\mathrm{IC}_{80 \%}\right)^{* *}$ & $\begin{array}{c}\text { p-value } \\
\text { (LR-test) }^{\text {L** }}\end{array}$ & $\mathrm{RP}^{* *+*}\left(\mathrm{IC}_{95 \%}\right)^{* *+}$ & $\begin{array}{c}p \text {-value } \\
\text { (LR-test) }^{\star * * *}\end{array}$ \\
\hline \multicolumn{5}{|l|}{ Via de nascimento } \\
\hline Cirurgia cesárea & 1 & $<0,001$ & 1 & 0,001 \\
\hline Vaginal & $1,47(1,32-1,63)$ & & $1,48(1,26-1,73)$ & \\
\hline \multicolumn{5}{|l|}{ Período da semana } \\
\hline Terça a sexta-feira & 1 & 0,001 & 1 & 0,001 \\
\hline Sábado a segunda-feira & $0,82(0,76-0,89)$ & & $0,83(0,74-0,94)$ & \\
\hline Escolaridade (em anos de estudo) & $1,02(1,01-1,03)$ & 0,005 & $1,02(1,01-1,03)$ & 0,012 \\
\hline \multicolumn{5}{|l|}{ Classificação do parto } \\
\hline Á termo & 1 & 0,009 & 1 & 0,008 \\
\hline Prematuro & $0,71(0,60-0,84)$ & & $0,70(0,55-0,91)$ & \\
\hline Tempo de permanência do acompanhante ao lado da mulher (horas) & $1,00(0,99-1,00)$ & 0,491 & $1,00(0,99-1,01)$ & 0,977 \\
\hline
\end{tabular}

"Razão de prevalência bruta; "Intervalo de confiança de 80\%; "'Teste de razão de verossimilhança; "'t"Razão de prevalência conforme modelo final ajustado 


\section{Discussão}

Nas maternidades da RMF mais de 70\% dos acompanhantes relataram algum tipo de VI contra a mulher durante a internação para o parto, tendo como fatores associados o parto vaginal, a termo, ocorrido entre terça e sexta-feira, relatada com mais frequência por acompanhantes com maior escolaridade. Até onde se sabe, este é o primeiro estudo sobre a VI contra a mulher durante o atendimento obstétrico a partir do relato do acompanhante.

O percentual de relato de acompanhante de pelo menos um tipo de violência contra a mulher durante a internação para o parto encontrado $(73,5 \%)$ é próximo do relatado por puérperas $(70 \%)$ em estudo nacional e países da África. ${ }^{(20-23)}$ A discriminação social é um dos determinantes da VI, mais frequente nas maternidades públicas, onde a maioria das usuárias pertence às classes sociais menos favorecidas. ${ }^{(8,24)}$

O parto vaginal é um fator de risco para a VI, comumente associado ao comportamento da parturiente - considerado inadequado pela equipe -, ao maior tempo de permanência na instituição e a construção sociocultural do parto como evento de dor e sofrimento. ${ }^{(25,26)} \mathrm{O}$ aumento na chance de relatos de VI por acompanhantes de parto vaginal, observado nas maternidades da RMF foi semelhante ao encontrado em outros estudos com puérperas em diferentes partes do mundo. . $^{(2,3,5,9,21,27)}$

$\mathrm{O}$ fato de acompanhantes de parto prematuro na RMF terem relatado menos VI contra a mulher, mostrando-se um fator de proteção, pode ser explicado pela gravidade da situação que demanda maior atenção dos prestadores de cuidado para evitar o pior desfecho e ao nervosismo e preocupação do acompanhante e da parturiente que dificultam a percepção da violência.

As queixas de pior atendimento em instituições hospitalares são mais frequentes aos finais de semana. ${ }^{(22)}$ Em discordância, nesse estudo, os acompanhantes relataram $17 \%$ menos violência durante o período de sábado a segunda-feira, o que pode ser explicado pelo maior fluxo de alunos de cursos da área da saúde durante os dias úteis. $\mathrm{O}$ volume de pessoas que interagem com a parturiente e seu acompanhante podem gerar situaçóes de violência, em especial no que se refere às falhas na comunicação.
O aumento na escolaridade do indivíduo amplia o acesso a informação e contribui para que ele reivindique seus direitos, como sugerem os achados do estudo. $\mathrm{O}$ acesso a informaçóes sobre a gestação, o parto e a internação, também auxiliam no empoderamento do acompanhante para identificar e combater a VI. ${ }^{(13,15,28)}$

A escolha do companheiro da mulher e pai do bebê como acompanhante do parto, foi observada em outros estudos. ${ }^{(15,16,29)}$ Diniz et al., ${ }^{(4)}$ em estudo nacional sobre a inserção de acompanhantes entre 2011 e 2012, demonstraram que as maternidades das regióes Sul e Sudeste $(22,6 \%$ e 23,1\%) tiveram melhores resultados. No entanto, os resultados desse estudo sugerem que ainda há desafios a serem enfrentados.

A elevada prevalência de relatos de VI estrutural pelo acompanhante de parto $(59,2 \%)$ provoca uma reflexão sobre a organização do serviço, dimensionamento de recursos humanos, nas rotinas assistenciais e na adequação da infraestrutura para o parto. A Resolução nº 36 da Agência Nacional de Vigilância Sanitária, em 2008, definiu as condiçóes mínimas para garantir o atendimento de qualidade e digno à parturiente. ${ }^{(30)}$ Mas, uma década depois, persistem graves violaçóes dos direitos humanos, como a perda de autonomia e práticas prejudiciais à integridade física e emocional da parturiente. ${ }^{(31)}$

A nota do Secretario Geral da Organização das Naçóes Unidas sobre o enfoque dos Direitos Humanos sobre maus tratos e violência contra as mulheres em serviços de saúde no momento do atendimento ao parto elaborada a partir da Relatoria Especial do Departamento de Direitos Humanos da ONU aponta, que no contexto dos serviços de saúde materna e reprodutiva as condiçóes e limitaçóes do sistema de saúde são causas subjacentes de maus tratos e da violência contra a mulher durante a atenção ao parto. ${ }^{(32)}$

Ademais, a manutenção de práticas violentas no atendimento obstétrico é legitimada pela cultura do parto baseada no uso excessivo de tecnologia e medicalização, no controle profissional e no intenso sofrimento que a acompanha na transição para a maternidade. ${ }^{(22,25,26,33)} \mathrm{O}$ inquérito nacional sobre a atenção obstétrica no Brasil mostrou que 65,5\% 
das mulheres que tiveram partos em maternidades entre 2011-2012, sofreram restrição de dieta e 55\% de mobilidade. ${ }^{34)}$

Os acompanhantes de parto em maternidades públicas da RMF, também relataram a VI física contra a parturiente com prevalência (30\%) que é próxima àquela encontrada em estudo com puérperas na Etiópia $(38,6 \%)$ e no Quênia (38\%). ${ }^{(21,22)}$ A semelhança desse resultado com os achados outros em países com grandes desigualdades sociais e poucas garantias de direitos, reforça a discriminação relacionada aos fatores sociais como determinante da VI no parto.

Estudos realizados com puérperas no Brasil, registraram prevalências menores de VI física. ${ }^{(20,35)}$ Essa discrepância entre as prevalências de relatos de acompanhante e de puérperas pode ser atribuída ao receio da mulher em denunciar a violência sofrida, por desconhecer seus direitos, e pela tendência da mulher em minimizar as situaçôes desagradáveis antecedentes ao nascimento, quando o desfecho é favorável. ${ }^{(31)}$ Embora o acompanhante, também esteja envolvido emocionalmente e muitas vezes fragilizado, ele é um agente externo, sendo capaz de relatar, de forma mais crítica os acontecimentos vivenciados. As diferenças metodológicas para a definição de VI física também podem ter contribuído para as diferença encontradas. ${ }^{(14,25,29)}$

A VI psicológica, relatada pelos acompanhantes (4,7\%), é uma das formas mais sutis e difíceis de identificar, por estar camuflada nas relaçóes sociais e nos significados simbólicos do parto, com repercussóes na vida da mulher. ${ }^{(4)} \mathrm{O}$ abandono e a negligência das necessidades da parturiente pelos profissionais são as situações mais frequentes desse tipo de violência, também associadas a discriminação social e de gênero. ${ }^{(21,27)}$

A prevalência da VI verbal relatada pelos acompanhantes nesse estudo foi inferior ao encontrado por Lansky e colaboradores ${ }^{(20)}$ (33\%) e por Mesenburg e colaboradores ${ }^{(35)}(10 \%)$, sugerindo que a presença do acompanhante pode reduzir esse tipo de violência, embora não seja suficiente para impedi-la. ${ }^{(4,15,17)}$

Em revisão sistemática recente da Biblioteca Cochrane realizada por Bohren e colaboradores (2019) apontam que dentre as várias atuaçóes do acompanhante para o beneficio em relação ao in- cremento da experiência da mulher com o próprio parto também está o fato de que este pode atuar como uma voz das mulheres no sentido de falar pela mulher quando necessário. ${ }^{(12)}$

Destaca-se como limitaçóes do estudo o fato de não ter sido planejado para estimar a VI contra a mulher durante a internação para o parto, produzindo uma medida indireta do desfecho. A disponibilidade de poucos estudos que tenham o acompanhante como sujeito da pesquisa restringe a comparação dos achados com outros cenários.

\section{Conclusão}

As prevalências de violência estrutural, física, psicológica e verbal contra a mulher durante o parto, relatadas pelo acompanhante na internação obstétrica, mostram que a sua presença não impede a ocorrência da VI. Os fatores associados demonstram que ainda se precisa avançar na implementação das práticas baseadas em evidência e na consolidação do protagonismo da mulher no parto nas maternidades públicas da RMF. Para isso, barreiras precisam ser transpostas, especialmente nas atitudes e nos discursos de profissionais, bem como, no processo de trabalho das instituiçóes. A elevada prevalência de relato de VI pelo acompanhante revela a lacuna existente entre a política de saúde e a realidade das maternidades brasileiras. Apesar dos dispositivos normativos para o atendimento obstétrico, persistem inadequaçóes estruturais, insuficiência de recursos humanos, manutenção de fluxos assistenciais ultrapassados, representando riscos para a saúde da mulher e do recém-nascido. São necessárias investigaçôes, mais amplas e com diferentes metodologias, para desvelar as potencialidades do acompanhante como agente de controle social da VI contra a mulher durante a internação para o parto, contribuindo para a qualidade e a segurança da assistência.

\section{Agradecimentos}

Este estudo é um subprojeto do projeto maior "A participação do acompanhante de escolha da mulher 
no pré-natal, trabalho de parto e parto no sistema de saúde público e suplementar", subsidiado pelo Conselho Nacional de Desenvolvimento Científico e Tecnológico (Edital Universal 14/2013).

\section{Colaborações}

Marrero L e Brüggemann OM foram responsáveis pela concepção, desenho metodológico, análise e interpretação dos dados. Junges CF participou da coleta de dados e revisão da versão de submissão. Scheneck CA e Costa R participaram da revisão da versão de submissão.

\section{Referências}

1. Starr P. Professionalization and public health: historical legacies, continuing dilemmas. J Public Health Manag Pract. 2009;15(6 Suppl):S26-30

2. Morton $\mathrm{CH}$, Henley MM, Seacrist M, Roth LM. Bearing witness: united States and Canadian maternity support workers' observations of disrespectful care in childbirth. Birth. 2018;45(3):263-74.

3. Bohren MA, Vogel JP, Tunçalp Ö, Fawole B, Titiloye MA, Olutayo AO, et al. Mistreatment of women during childbirth in Abuja, Nigeria: a qualitative study on perceptions and experiences of women and healthcare providers. Reprod Health. 2017;14(1):9.

4. Diniz CS, d'Orsi E, Domingues RM, Torres JA, Dias MA, Schneck CA, et al. Implementation of the presence of companions during hospital admission for childbirth: data from the Birth in Brazil national survey. Cad Saude Publica. 2014;30(Supl): 140-53.

5. Organização Mundial da Saúde (OMS). Prevenção e eliminação de abusos, desrespeito e maus-tratos durante o parto em instituições de saúde. Genebra: OMS; 2014.

6. Sen G, Reddy B, lyer A. Beyond measurement: the drivers of disrespect and abuse in obstetric care. Reprod Health Matters. 2018;26(53):618

7. Sando D, Abuya T, Asefa A, Banks KP, Freedman LP, Kujawski S, et al. Methods used in prevalence studies of disrespect and abuse during facility-based childbirth: lessons learned. Reprod Health. 2017; 14(1):127.

8. Leal MD, Szwarcwald CL, Almeida PV, Aquino EM, Barreto ML, Barros F, et al. Reproductive, maternal, neonatal and child health in the 30 years since the creation of the Unified Health System (SUS). Cien Saude Colet. 2018;23(6):1915-28.

9. Venturi G, Bokany V, Dias R. Mulheres brasileiras e gênero nos espaços público e privado. São Paulo: Fundação Perseu Abramo; 2010.

10. Junges CF, Brüggemann OM, Knobel R, Costa R. Support actions undertaken for the woman by companions in public maternity hospitals. Rev Lat Am Enfermagem. 2018;26(0):e2994.

11. Teixeirense MM, Santos SL. From expectation to experience: humanizing childbirth in the Brazilian National Health System. Interface
Comun Saude Educ. 2018;22(65):399-410.

12. Bohren MA, Berger BO, Munthe-Kaas H, Tunçalp Ö. Perceptions and experiences of labour companionship: a qualitative evidence synthesis. Cochrane Database Syst Rev. 2019 Mar;3:CD012449.

13. Brüggemann OM, Koettker JG, Velho MB, Monguilhott JJ, Monticelli M. Monticelli Satisfaction of companions with the experience of supporting the parturient at a university hospital. Texto Contexto Enferm. 2015;24(3):686-96.

14. Souza KR, Dias MD. Oral History: experience of doulas in the care of women. Acta Paul Enferm. 2010;23(4):493-9.

15. Batista BD, Brüggemann OM, Junges CF, Velho MB, Costa R. Factors associated with the birth companion's satisfaction with the care provided to the parturient woman. Cogitare Enferm. 2017;22(3):4553.

16. Dulfe PA, Lima DV, Alves VH, Rodrigues DP, Barcellos JG, Cherem EO. Presence of a companion of the woman's choice in the process of parturition: repercussions on obstetric care. Cogitare Enferm. 2016;21(4):1-8.

17. Souza SR, Gualda DM. The experience of women and their coaches with childbirth. Texto Contexto Enferm. 2016;25(1):1-9.

18. Vasconcellos MT, Silva PL, Pereira AP, Schilithz AO, Souza Junior PR, Szwarcwald CL. Desenho da amostra Nascer no Brasil: Pesquisa Nacional sobre Parto e Nascimento. Cad Saude Publica. 2014;30 Supl. 1:S49-58.

19. Cribari-Neto F, Galvão NMS. A class of improved heteroskedasticityconsistent covariance matrix estimators. Commun Stat. 2003; 32(10):1951-80.

20. Lansky S, Souza KV, Morais ER, Oliveira BF, Diniz S, Vieira NF, et al. Violência obstétrica: influência da exposição sentidos do nascer na vivência das gestantes. Cien Saude Coletiva; 2018; 24(8):2811-23.

21. Asefa A, Bekele D. Status of respectful and non-abusive care during facility-based childbirth in a hospital and health centers in Addis Ababa, Ethiopia. Reprod Health. 2015;12(1):1-9.

22. Abuya T, Ndwiga C, Ritter J, Kanya L, Bellows B, Binkin N et al. The effect of a multi-component intervention on disrespect and abuse during childbirth in Kenya. BMC Pregnancy Childbirth. 2015;15:224.

23. Sethi R, Gupta S, Oseni L, Mtimuni A, Rashidi T, Kachale F. The prevalence of disrespect and abuse during facility-based maternity care in Malawi: evidence from direct observations of labor and delivery. Reprod Health. 2017;14(1):111.

24. Nakano MS, Silva LA, Beleza AC, Stefanello J, Gomes FA. 0 suporte durante o processo de parturição: a visão do acompanhante. Acta Paul Enferm. 2007;20(2):131-7.

25. Castrillo B. Dime quién lo define y te diré si es violento. Reflexiones sobre la violencia obstétrica. Sex Salud Soc. 2016; (24):43-68.

26. Sadler M, Santos MJ, Ruiz-Berdún D, Rojas GL, Skoko E, Gillen $P$, et al. Moving beyond disrespect and abuse: addressing the structural dimensions of obstetric violence. Reprod Health Matters. 2016;24(47):47-55.

27. d'Orsi E, Brüggemann OM, Diniz CS, Aguiar JM, Gusman CR, Torres JA, et al. Social inequalities and women's satisfaction with childbirth care in Brazil: a national hospital-based survey. Cad Saude Publica. 2014;30 Suppl 1:S1-15.

28. Teles LM, Américo CF, Oriá MO, Vasconcelos CT, Brüggemann OM, Efficacy of an educational manual for childbirth companions: pilot study of a randomized clinical trial. Rev Lat Am Enfermagem. 2017; 25:e2996. 
29. Monguilhott JJ, Brüggemann OM, Freitas PF, d'Orsi E. Nascer no Brasil: the presence of a companion favors the use of best practices in delivery care in the South region of Brazil. Rev Saude Publica. 2018:52:1-11.

30. Agência Nacional De Vigilância Sanitária (ANVISA). Diretoria Colegiada. Resolução no. 36 de 3, de junho de 2008. Dispõe sobre Regulamento Técnico para Funcionamento dos Serviços de Atenção Obstétrica e Neonatal. [Internet]. Diário Oficial da União: seção 1: Poder Executivo, Brasília (DF):ANVISA; 2008. p.50. [citado 2018 Jan 2]. Disponível em: https://www20.anvisa.gov.br/segurancadopaciente/index.php/ legislacao/item/rdc-n-36-de-03-de-junho-de-2008.

31. Portela MC, Reis LG, Martins M, Rodrigues JL, Lima SM. Obstetric care: challenges for quality improvement. Cad Saude Publica. 2018;34(5):e00072818.
32. United Unions. Special Rapporteur on violence against women, its causes and consequences [Internet]. Geneva: United Unions; 2019 [cited 2019 Sep 16]. Available in: https://www.ohchr.org/en/issues/ women/srwomen/pages/srwomenindex.aspx

33. d'Oliveira AF, Diniz SG, Schraiber LB. Violence against women in healthcare institutions: an emerging problem. Lancet. 2002;359(9318):1681-5.

34. Baldisserotto ML, Theme Filha MM, da Gama SG. Good practices according to WHO's recommendation for normal labor and birth and women's assessment of the care received: the "birth in Brazil" national research study, 2011/2012. Reprod Health. 2016;13(S3 Suppl 3):124.

35. Mesenburg MA, Victora CG, Jacob SS, Ponce de León R, Damaso AH, Domingues MR. Disrespect and abuse of women during the process of childbirth in the 2015, Pelotas birth cohort. Reprod Health. 2018;15(1):54. 